\title{
INFLUENCE OF TOOL DIAMETER ON QUALITY OF COMPLETED RADIO AREA
}

\author{
Lubos Kroft, Jan Hnatik \& Katerina Bicova
}
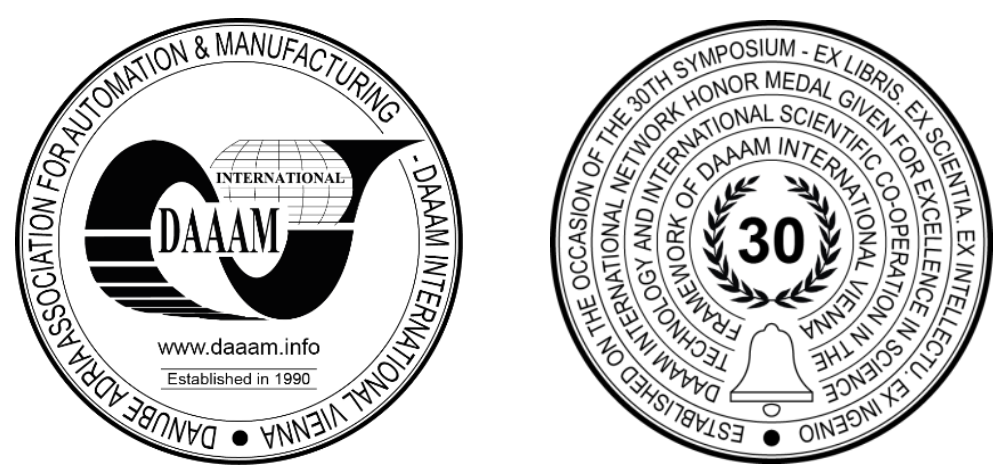

This Publication has to be referred as: Kroft, L[ubos]; Hnatík, J[an] \& Bicova, K[aterina] (2019). Influence of Tool Diameter on Quality of Completed Radio Area, Proceedings of the 30th DAAAM International Symposium, pp.05620569, B. Katalinic (Ed.), Published by DAAAM International, ISBN 978-3-902734-22-8, ISSN 1726-9679, Vienna, Austria

DOI: 10.2507/30th.daaam.proceedings.076

\begin{abstract}
The aim of this paper is to introduce one of the directions of research focused on finishing milling in CAD / CAM systems. This research is focused on this area since current finishing operations do not include any of the modern methods of tool path generation, eg through adaptive control or tool load control. The first part of this research focused on radius surfaces milling using a ball end mill is the input experiment of the whole research. Although the radius surface is a great simplification of the general shape surface, it is suitable for the first research in this area due to the continuous change of engagement conditions. The aim of this section is to describe the relationship between tool diameter and radius surface radius. The relationship is demonstrated by the achieved quality of the finished surface.
\end{abstract}

Keywords: Finishing milling; tool radius; roughness of finished surface; ball end mill.

\section{Introduction}

Finishing of freeform 3D surfaces can be performed using various machining processes. Milling is the most widelyused technology in this context. NC machines with CNC control systems are used for this purpose. As a result, various software tools for production support and optimisation take a prominent position, becoming indispensable for designing and developing machining strategies.

This concept of "strategy" underwent major changes in recent years. It used to denote the choice of a suitable machine tool or direction of machining. Today, a "strategy" refers to a function of a particular software tool. The advancement of technology enabled new processes to be deployed, such as HSC, HPC, hard machining, trochoidal machining and others. They led to shorter production times, longer tool life, better productivity and optimization of the entire process. Yet, these advanced finishing strategies are used rarely.

This paper aims to present research into finishing operations in advanced CAM systems. As this involves many pitfalls, the focus is on exploring the impact of the choice of finishing strategy and tool diameter on the process of finishing of round surfaces. [1],[8] 


\section{Machining of round surfaces}

The process chain for operations planning can be denoted as CAD/CAM/CNC. Despite advanced production capabilities, machining of 3D sculptured surface still poses numerous difficulties.

A round surface is a simple example of a shaped surface. Although the transition from level to level involves no more than two-dimensional change, it is useful for exploring different strategies in CAM system for multiple reasons. First, one can control the engagement conditions, the arc of engagement and other cutting parameters. It is also well-suited for measurement and evaluation. Another favourable aspect is that the point of contact shifts continuously within the range of angles $0^{\circ}-90^{\circ}$.

Considering the expected output from this research, a round surface is a convenient simplification of a freeform surface. Round surfaces find use in most industrial sectors. They are found on rotating parts as well as on non-rotating ones. The range of sectors of their application includes the automotive and aviation industries, healthcare and power generation.

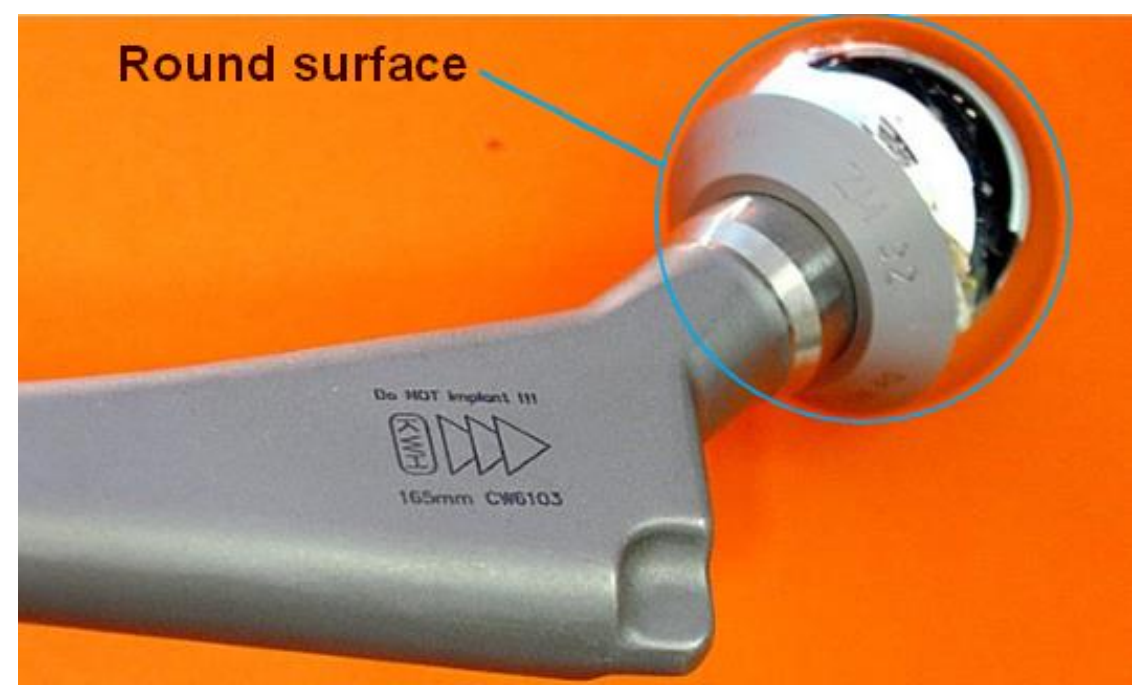

Fig. 1. - Example of a round surface [7]

A round surface can be understood as a shaped surface having certain radius $\mathrm{R}$ - particularly in those cases when it is to be manufactured without rotating the workpiece, e.g. by milling. Freeform surfaces created with splines can also be considered to be connected round surfaces. For all these reasons, machining of round surfaces is a relevant and frequentlyencountered task. The purpose and size of a round surface are typically governed by the function of the component. In rare cases, it is part of a decorative item.

\section{Design of an experiment for finishing round surfaces}

One of the available approaches to finishing involves removal of constant volumes of material. It is derived from roughing strategies, where the toolpath for the round surface was generated in a way which ensures that near constant volumes of material are removed. This approach has not yielded any clear benefits. Advantages of constant volume removal only became appreciable when the allowance for finishing was much larger than the standard amount. In that case, toolpath adjustment based on removal volume led to reduced cutting forces. It improved stability of the cutting process, mainly in those locations where the workpiece was engaged by the portions of the tool close to its axis. [2]

Hence, this part of the research focused on the diameter of finishing tools. Finishing was performed with a ball mill. The relationship between the tool diameter and the finished surface was studies. In addition, the effects of the different machining strategies were explored.

\subsection{Theoretical foundations of finishing of round surfaces}

The fundamental attribute of finishing freeform surfaces with a ball end mill is the variable point of contact between the tool and the workpiece (CC - Cutter Contact). It is a point on a cutting edge of the tool which is in contact with the work. Using the location of this point, one can derive the effective diameter of the tool $\mathrm{D}_{\text {ef. }}$. As the slope of the tangent at $\mathrm{CC}$ varies across a 3D freeform surface, the value of $\mathrm{D}_{\text {ef }}$ varies by the same token, as shown in Fig. 2. [1] 

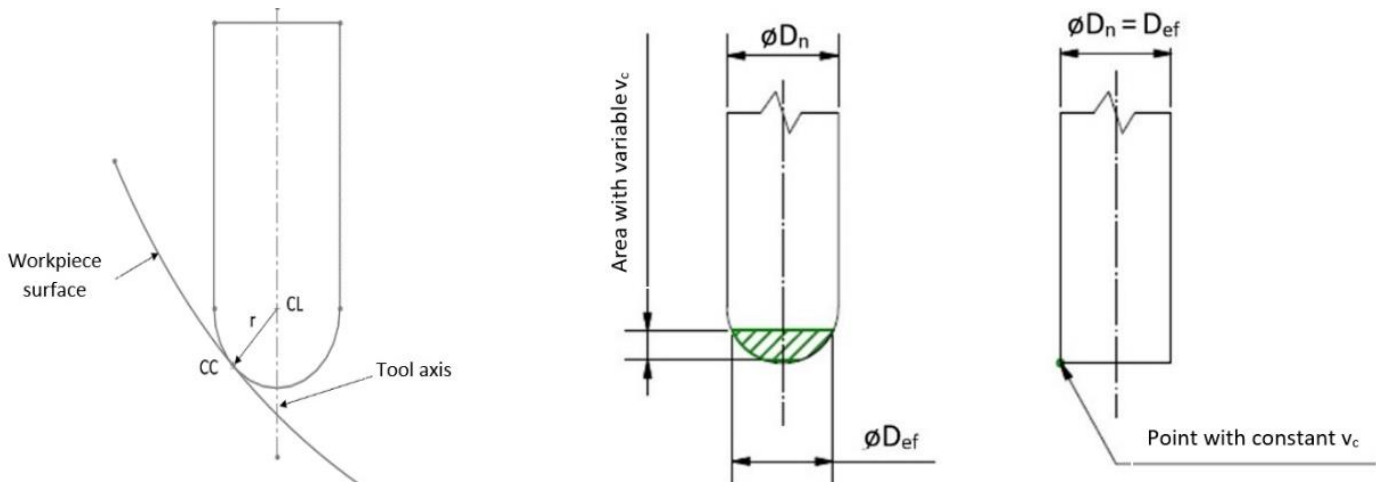

Fig. 2. - Position of CC on a freeform surface and effective diameters for a ball mill and an end mill [4]

In CAM tools, speeds are calculated in the standard strategies on the basis of the maximum diameter of the tool Dn. These speeds are then applied to all path under the strategy. However, as Def varies, cutting speed varies as the tool moves across the shaped surface. Different diameters and the region of non-constant vc are shown in Fig. 2.

This relationship has a number of negative consequences. One of them is that several different finishing strategies need to be combined to machine sculptured parts, which involves a number of difficulties. Changes in cutting conditions make it difficult to achieve uniform quality of the finished surface. These and other aspects often make CAM users adjust the resulting toolpaths to avoid these drawbacks. In practice, finishing toolpaths are often generated from several different strategies available in the CAM tool, becoming denser or involving pre-finishing operations, which hurts efficiency and drives up production costs.

\subsection{Output parameters}

The output parameters for evaluation include roughness and dimensional accuracy of the finished surface, i.e. its overall quality. Cutting forces were measured in the process. There are a number of surface roughness parameters. Those which were measured in the experiment are below:

- Cutting force components Fxy and F

- Surface roughness parameters $\mathrm{Ra}$ and $\mathrm{Rz} \mathrm{Rz}$

- Dimensional accuracy evaluated from deviation of points on the surface from the part's model

\subsubsection{Cutting forces}

Cutting forces which occur as the work material is removed are affected by many aspects. Generally, the highest values of cutting forces encountered during finishing cannot be seen as indicators of overall safety and stability of the process. The forces in the finishing stage do not reach values which arise in rough milling or drilling. Therefore, one cannot assess finishing process by the highest cutting force.

What is important in cutting forces during finishing is their overall profile. Fluctuations, which may amount to dozens of percent, may cause vibrations or variation in tool displacement, leading to non-uniform surface quality. Cutting forces were therefore measured as complementary data. If variations are found in other parameters, these data may help explain the results.

Since analyzing individual components Fx, Fy and Fz would probably fail to provide explanations of the expected links to qualitative parameters, a scalar product in XY plane was used for the final evaluation. The values of cutting forces Fxy are related to tool displacement and should correlate with some of the qualitative parameters. Another parameter under evaluation is total cutting force $\mathrm{F}$, as it incorporates all the components measured.

\subsubsection{Surface roughness}

Cutting a surface by an appropriately-oriented hypothetical plane yields a curve consisting of peaks and valleys. This curve is a representation of the surface texture. Although there is a variety of surfaces, their measurement methods and in particular their quality evaluation, the key parameter for evaluating this curve is roughness. [5]

The overall roughness of a milled surface is a result of a number of factors. Roughness values may strongly depend on the relative direction of the cutting tool and measurement. Values measured parallel to the machining direction will be 
strongly influenced by the feed rate. Those measured perpendicularly to the machining direction will be affected by stepover and tool size. For these reasons, the present study involved measurement in both directions.

For the result itself and its representation, it is also important to use a suitable software filter for evaluation as reported by some sources, eg an article from the source [6]. For our case with regard to the considered deployment of the obtained results on the general shape surface, a standard Gaussian filter according to EN ISO 16610-21 was used, which is suitable for the given application according to the available source.

\subsubsection{Dimensional accuracy}

The last parameter reviewed was the dimensional accuracy of the finished surface. In order to be able to compare the measured results with at least a portion of any data available in the future, one should choose a method which is applicable to freeform surfaces. For ranking the factors and variants, the output had to be quantifiable.

Based on all these criteria, a 3D coordinate measuring machine was chosen for determining the dimensional accuracy of the surface. Deviations of points of the surface from the model were measured. The surface of the model was the reference from which deviations in relevant locations were measured.

\section{Experimental procedure}

Three variable factors related to the effects in question were chosen for designing this experiment. The first factor was the radius of the surface being finished. As shown in the figure 3, a round surface with a slope ranging from $0^{\circ}$ to $90^{\circ}$ was chosen. Across this surface, the slope of the tangent line at the CC contact point varies continuously, which enables us to explore the effect of this variation on final roughness produced with different strategies. In order to obtain data for general conclusions, round surfaces with $\mathrm{R}=10-50 \mathrm{~mm}$ were chosen.

Another factor was the choice of the stepover calculation strategy.

The last variable factor was the tool diameter. Its impact on the quality of the finished surface was studied. This relationship is well known: lower roughness is normally obtained with larger-diameter tools. A majority of experimental reports, however, only deal with a single tool diameter value. Therefore, no descriptions of the effect on the finished surface quality was found for either round or freeform surfaces.
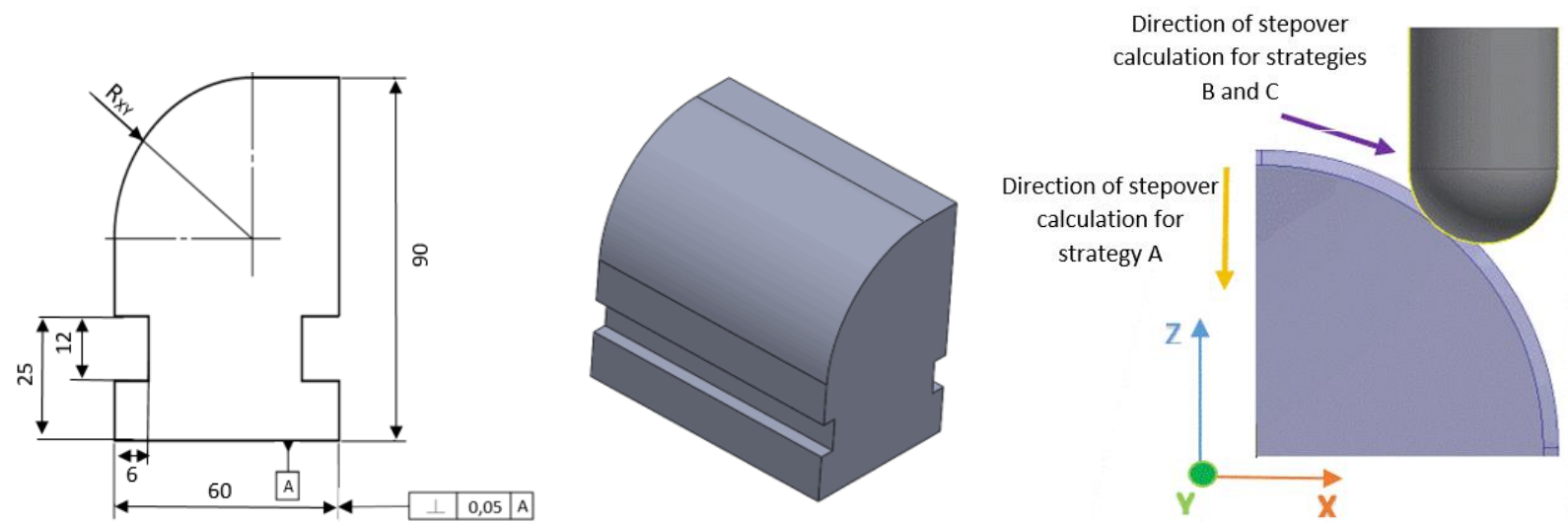

Fig. 3. - Experimental specimen and stepover calculation directions for individual strategies and specimens indicated with respect to the coordinate system of the machine [4]

Three fundamental toolpath stepover calculations available in almost all CAM systems today: stepdown along an axis, stepover along a curve and stepover based on rest material. In this case, the axis used for stepdown calculation was the tool axis. Calculation strategies along a curve and on the basis of rest material relied on a secant line to the radius for stepover calculation. The curve-based calculation used a pre-defined stepover value, whereas the other involved calculation of instant stepover values based on rest material. All directions are illustrated in Fig. 3.

The full design of the experiment is illustrated in Table 1. 


\begin{tabular}{|c|c|c|c|c|c|c|}
\hline \multirow{3}{*}{$\begin{array}{l}\text { Strategy } \\
\text { Radius of the surface being } \\
\text { finished [mm] }\end{array}$} & \multirow{2}{*}{\multicolumn{2}{|c|}{$\begin{array}{c}\text { A - constant } \\
\text { stepdown along } \mathrm{Z} \\
\text { axis }\end{array}$}} & \multirow{2}{*}{\multicolumn{2}{|c|}{$\begin{array}{l}\text { B - constant stepover } \\
\text { along a curve }\end{array}$}} & \multirow{2}{*}{\multicolumn{2}{|c|}{$\begin{array}{c}\mathrm{C}-\text { stepover based on } \\
\text { rest material }\end{array}$}} \\
\hline & & & & & & \\
\hline & $\mathrm{R}$ & 10.201 & 16 & 30 & 44 & 49.799 \\
\hline Milling cutter diameter [mm] & $\mathrm{D}_{\mathrm{n}}$ & 3 & 4.9 & 9.5 & 14.1 & 16 \\
\hline \multicolumn{7}{|l|}{ Fixed factors } \\
\hline Feed per tooth [mm] & $\mathrm{f}_{\mathrm{z}}$ & & & 0.1 & & \\
\hline Radial stepover [mm] & $a_{e}$ & & & 0.1 & & \\
\hline Rest material thickness [ $\mu \mathrm{m}]$ & & & & 0.9 & & \\
\hline Cut depth [mm] & $a_{p}$ & & & 0.2 & & \\
\hline Cutting speed [m/min] & $\mathrm{V}_{\mathrm{c}}$ & & & 80 & & \\
\hline Number of teeth [-] & of & & & 4 & & \\
\hline
\end{tabular}

Table 1. Input factors for experiment [1]

\subsection{Test specimen}

The design of the test specimen was based on the standard shape suitable for mounting into a load cell. It was a prism of $60 \times 100 \times 90 \mathrm{~mm}$ size. Schematic depiction of the specimen, including basic dimensions and its 3D model, are shown in Fig. 3. The specimens were made of standard DIN C45 steel.

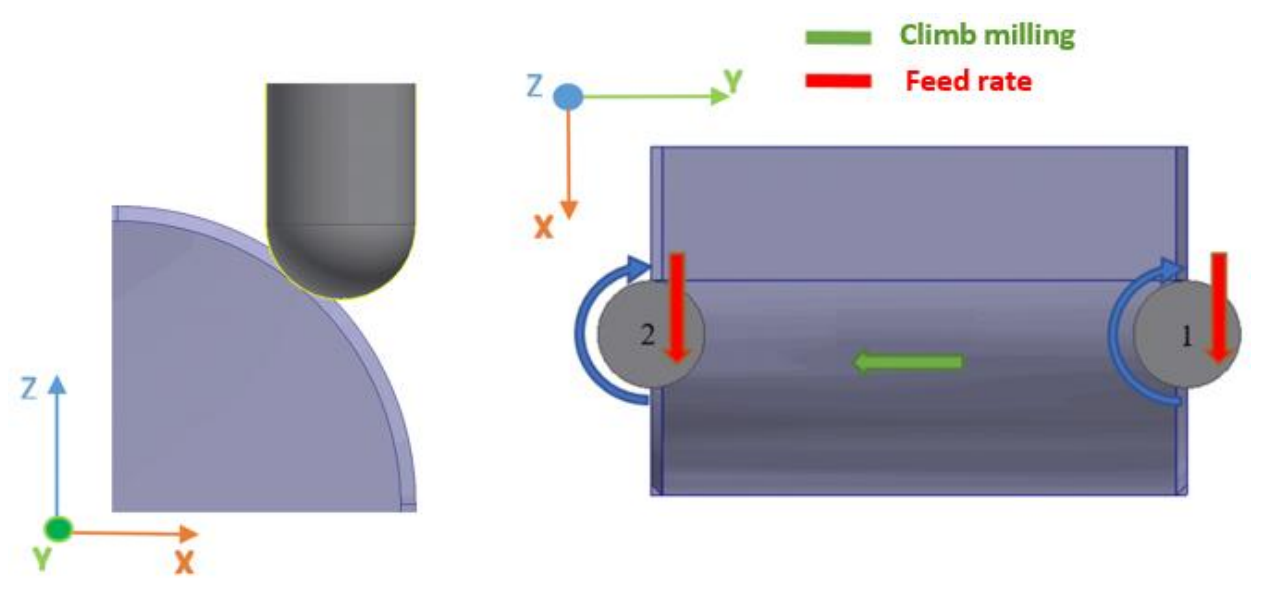

Fig. 4. - Toolpath directions on round surface [4]

In order to provide uniform conditions for finishing, the specimen was roughed and pre-finished to leave an allowance of $0.2 \mathrm{~mm}$ on the functional surface. The direction of finishing is shown in Fig. 4.

\section{Analysis of results of experiment}

As the amount of measured data was substantial, the evaluation comprised several stages. The single most important aspect is the surface parameters.

Among them, the key to the evaluation of the experiment were the qualitative ones. For finishing operations, surface roughness is more relevant, as the parameter which is monitored more often in freeform surfaces. Dimensional accuracy is difficult to assess on sculptured surfaces, which makes this parameter less relevant for the evaluation. The least emphasis was attached to cutting forces.

Of all the factors which impact the roughness of the finished surface, the dominant one was the choice of the toolpath and stepover calculation strategy. Measurements of the specimen showed that the right strategy leads to much lower and more uniform roughness. 


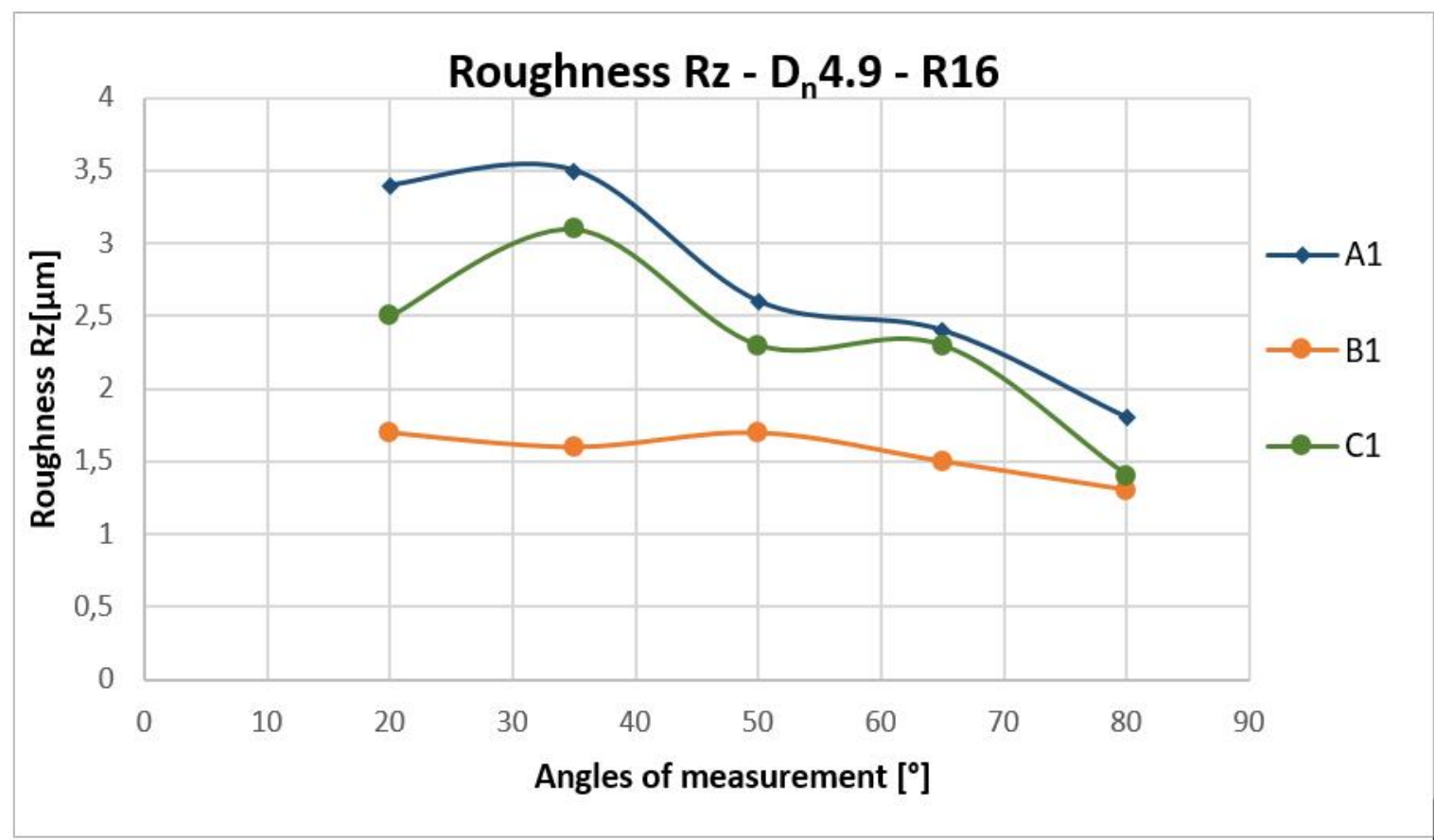

Fig. 5. - Roughness Rz plot for individual strategies on the surface with $\mathrm{R}=16 \mathrm{~mm}$ machined with a $\mathrm{Dn}=4.9 \mathrm{~mm}$ tool

Similar outcomes were obtained for average values from all the test specimens. Here, the average for strategy B was the highest but the range was the narrowest.

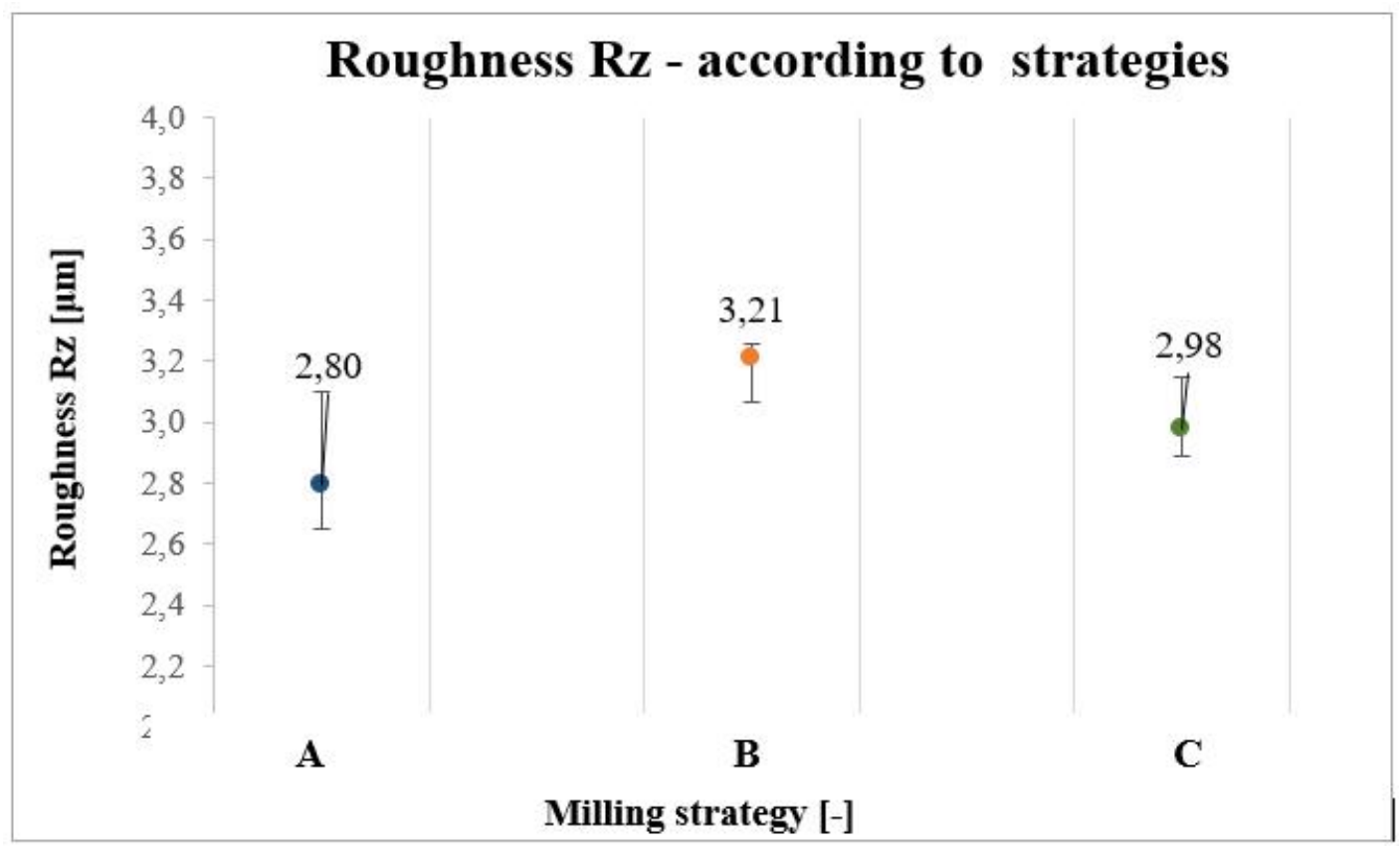

Fig. 6. - Graph of mean values and variance for roughness $\mathrm{Rz}$ - according to strategies [3]

The effects of the tool diameter were in line with machining theory. Larger-diameter tools produce surfaces with lower roughness. Changes in the radius of the surface had no adequately demonstrable impact on the roughness of the finished surface.

Evaluation of dimensional accuracy brought no significant results for any variable factor. The stepover calculation affects the deviation of the surface from the ideal surface but the effect cannot be demonstrated in this evaluation. It is only clearly visible from average values. Tool diameter and surface radius factors led to no relevant results. 


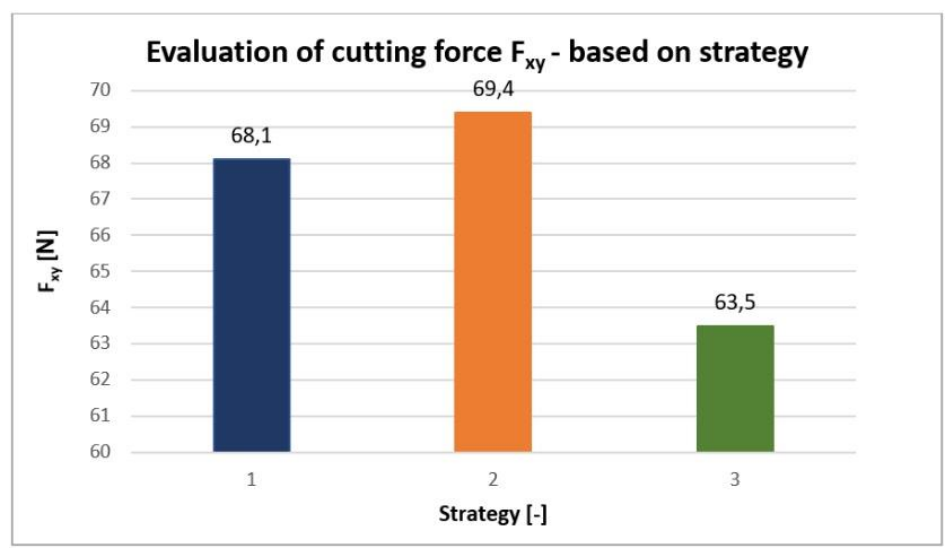

Fig. 7. - Graphical comparison of average values of cutting force Fxy [1]

Investigation into cutting forces revealed the impact of the toolpath and stepover calculation strategy. By selecting an appropriate strategy, one can obtain lower cutting forces, namely in XY plane.

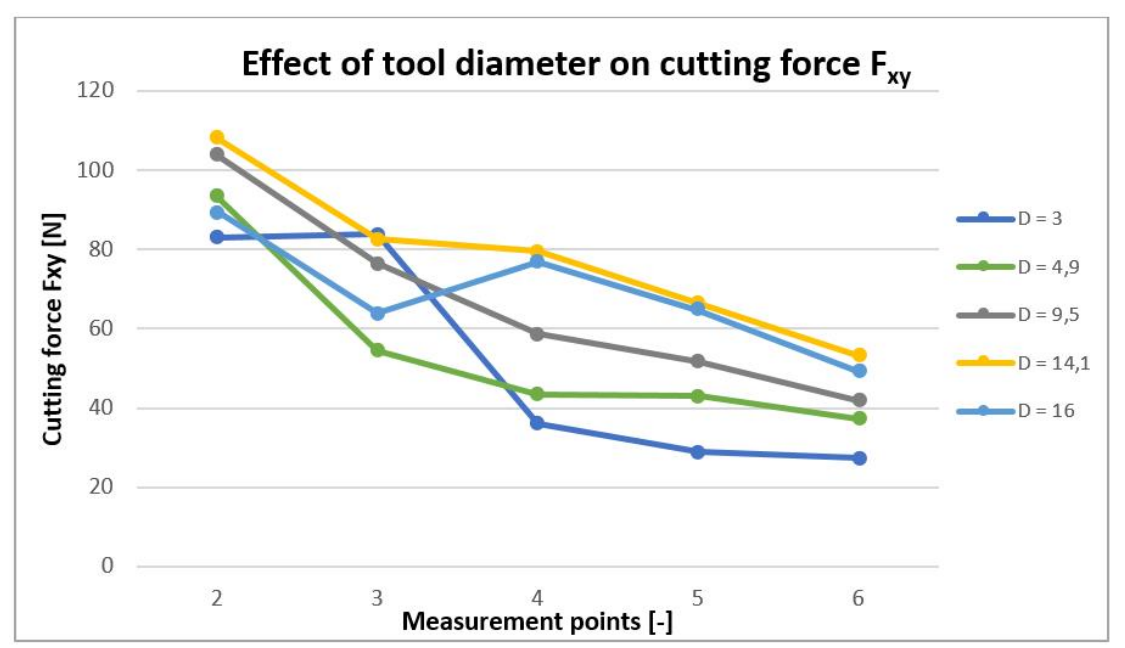

Fig. 8. - Effect of tool diameter on cutting force Fxy [3]

The effect of the tool diameter on cutting force profile is only visible above the $35^{\circ}$ angle of the tangent at the contact point. With increasing diameter, the cutting force increases; by no more than several dozen newtons. By contrast, under $35^{\circ}$, the effect of the surface radius can be observed. In this region, higher cutting forces are recorded on larger-radius surfaces and vice versa. Correlation between these results and other parameters were not found as the results were not conclusive.

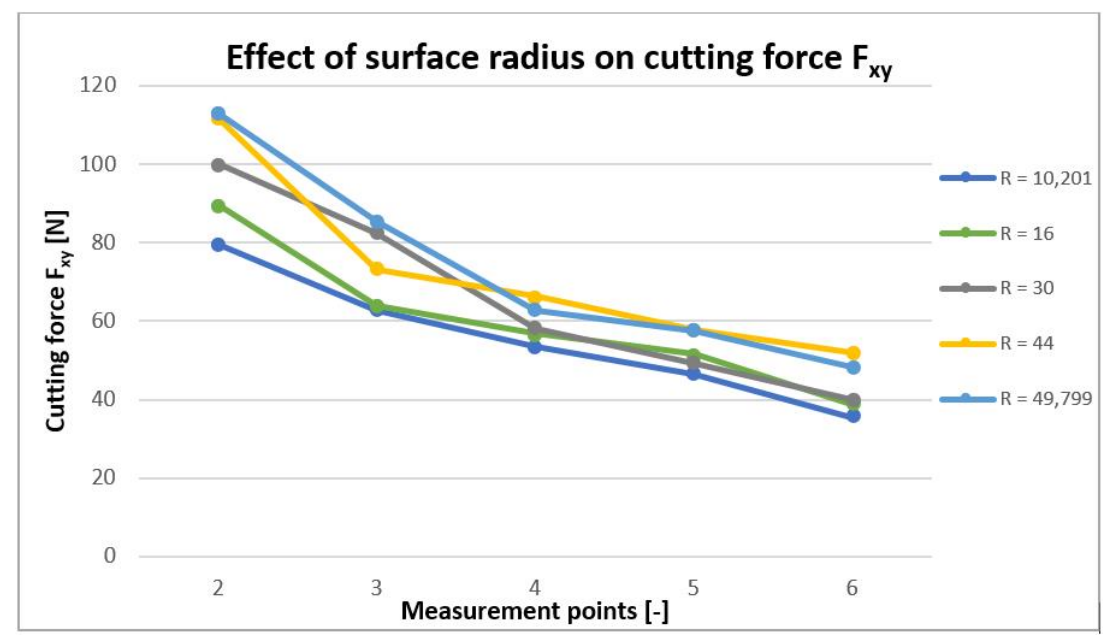

Fig. 9. - Effect of surface radius on cutting force Fxy [4] 
For some points, the experimental results provide clear information on the impact of a factor on the round surface being finished. It is particularly the choice of the stepover calculation strategy which can lead to better roughness.

With the other two parameters, the cutting forces and dimensional accuracy, the results are ambiguous. Understandably, any strategy needs to be verified by experiments prior to actual deployment, particularly with sculptured surfaces and different structural materials.

\section{Conclusion}

This paper aims to present research into finishing operations in advanced CAM systems. As this involves many pitfalls, the focus is on exploring the impact of the choice of finishing strategy and tool diameter on the process of finishing of round surfaces.

Unlike previous tests and experiments reported in literature, the present investigation focused on the interrelationship between the surface radius and the tool diameter. The motivation for this was the need to develop a methodology of design of finishing strategy under a research project. Results of the second experiment proved the favourable impact of machining strategy on surface roughness. With larger-diameter tools, lower and more uniform surface roughness can be achieved.

Future efforts in developing finishing strategies should incorporate additional principles which improve machining efficiency, e.g. adaptive generation of toolpath. They should be verified in machining of various materials and sculptured freeform surfaces. Combinations of various principles should be validated. Another potential theme involves the use of 3D corrections and splitting their applicability across different region of the surface, as opposed to their uniform application throughout a strategy.

\section{Acknowledgments}

This article was created under the project SGS-2019-008: Research and Development for Innovation in the Field of Manufacturing Technology - Machining Technology III.

\section{References}

[1] Kroft, L. (2018) The optimization machining strategies of the complex spatial areas, Dissertation thesis, Faculty of Mechanical Engineering - Technology of Metal Cutting, University of West Bohemia, Pilsen, Czech Republic.

[2] Hnatik, J; Kroft, L. \& Kutlwaser, J. (2016). Constant Chip Volume Machining, Proceedings of the 26th DAAAM International Symposium, pp.0960-0966, B. Katalinic (Ed.), Published by DAAAM International, ISBN 978-3902734-07-5, ISSN 1726-9679, Vienna, Austria DOI: 10.2507/26th.daaam.proceedings. 135

[3] Franěk, T. (2018) Influence of tool diameter on finish quality of radius surface. Diploma thesis, Faculty of Mechanical Engineering - Technology of Metal Cutting, University of West Bohemia, Pilsen, Czech Republic

[4] Jásek, O. (2018) Influence of cutting speed change on shape accuracy of finished surface. . Diploma thesis, Faculty of Mechanical Engineering - Technology of Metal Cutting, University of West Bohemia, Pilsen, Czech Republic

[5] Pospěch, Z. Lectures from workshop metrology - Surface roughness. Internal source of Faculty of Mechanical Engineering of University of West Bohemia

[6] Kubatova, D.; Melichar, M. \& Kutlwaser, J. (2018).Methodology for Selecting Software Filters, Proceedings of the 29th DAAAM International Symposium, pp.1266-1272, B. Katalinic (Ed.), Published by DAAAM International, ISBN 978-3-902734-20-4, ISSN 1726-9679, Vienna, Austria DOI: 10.2507/29th.daaam.proceedings. 182

[7] Leonardo technology. Laser marking SOLARIS. [Online] [reference: $27 . \quad$ October 2017.] http://www.lt.cz/produkty/laserove-znaceni/vlaknovy-laser/e-solarmark-fls.

[8] Zou, Q.; Zhang, J.; Deng, J. \& Zhao, J. Iso-level tool path planning for free-form surfaces [online]. [ref. $2018-07-$ 03]. DOI: 10.1016/j.cad.2014.04.006. ISBN $\quad 0010-4485 . \quad$ Available from: http://linkinghub.elsevier.com/retrieve/pii/S0010448514000700. 